\title{
Assessment of serological markers of dengue and it's correlation to thrombocytopenia Running title: Platelet counts and serological markers of dengue infection
}

\author{
Goel N. ${ }^{1}$, Kansal R. ${ }^{2 *}$, Joon A. ${ }^{3}$ \\ DOI: https://doi.org/10.17511/jopm.2020.i05.02
}

1 Neha Goel, Assistant Professor, Department of Microbiology, GS Medical College and Hospital, Hapur, Uttar Pradesh, India.

2* Ritika Kansal, Assistant Professor, Department of Pathology, GS Medical College and Hospital, Hapur, Uttar Pradesh, India.

3 Amit Joon, Assistant Professor, Department of Community Medicine, GS Medical College and Hospital, Hapur, Uttar Pradesh, India.

\begin{abstract}
Background: Dengue is an acute, potentially fatal viral infection that can culminate in dengue hemorrhagic fever and dengue shock syndrome. The present study was conducted to assess the correlation between platelet counts and serological markers of dengue infection. Materials and Methods: The present study was conducted among 120 patients suspected of dengue fever. Serum samples were collected from suspected dengue fever patients. The samples were tested for NS1 antigen, IgM, and IgG antibodies using the ICT test kit. Results: Out of 120 patients, males were 70 and females were 50 . NS1 only was detected in 62 , IgM only in 20, IgG only in 14, NS1 and IgM only in 10, NS1 and IgG only in 8 and IgM and IgG only in 6 cases. 62 patients that were positive for NS 1 , thrombocytopenia was seen in 48,12 out of $20 \mathrm{IgM}$ positive patients, 10 out of $14 \mathrm{IgG}$ positive patients, 6 out of 10 NS1 and IgM only positive patients, 4 out of 8 NS1 and IgG only positive patients and 2 out of $6 \mathrm{IgM}$ and IgG only positive patients were detected. Conclusion: In fever, thrombocytopenia is observed more uniformly in subjects positive for dengue. WheneverIgM is detected as compared to NS1 and can be used asan indicator to reduce the complication of dengue disease.
\end{abstract}

Keywords: Dengue, Serological markers, Thrombocytopenia

Corresponding Author

Ritika Kansal, Assistant Professor, Department of Pathology, GS Medical College and Hospital, Hapur, Uttar Pradesh, India.

Email: dr.ritikakansal@gmail.com
How to Cite this Article

Goel N, Kansal R, Joon A. Assessment of serological markers of dengue and it's correlation to thrombocytopenia Running title: Platelet counts and serological markers of dengue infection. Trop J Pathol Microbiol. 2020;6(5):362-365.

Available From

https://pathology.medresearch.in/index.php/jopm/ar ticle/view/463
To Browse

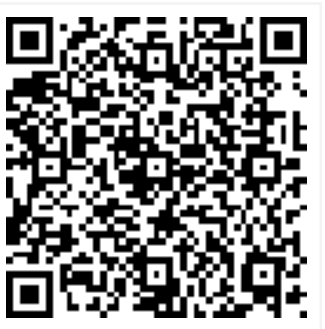

\section{Introduction}

>Dengue is an acute, potentially fatal viral infection that can culminate in dengue hemorrhagic fever (DHF) and dengue shock
Syndrome (DSS). It is caused by four serotypes of dengue virus (DV), namely DEN1, DEN-2, DEN-3, and DEN-4 belonging to genus Flavivirus and family Flaviviridae [1]. It is spread through the bite of infected
Manuscript Received 26-05-2020

Conflict of Interest No

(c) 2020 by Neha Goel, Ritika Kansal, Amit Joon and Published by Siddharth Health Research and Social Welfare Society. This is an Open Access article licensed under a Creative Commons Attribution 4.0 International License

Review Round 1 07-06-2020

Funding Nil
unding 12-06-2020 https://creativecommons.org/licenses/by/4.0/ unported [CC BY 4.0].

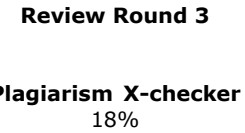

Review Round 3

lagiarism $\mathbf{X}$-checker $18 \%$

Accepted $17-06-2020$

Note 
Aedesaegypti mosquito. Most primary infections are uneventful. Infection with one serotype confers an individual life-long immunity to that serotype and cross-reactivity to the other serotypes [2]. The complications like DHF and DSS are usually attributed to this cross-reactivity. Dengue is almost endemic throughout India. The resource-poor health care system has to depend upon simple to perform and easy to interpret laboratory tests for diagnosis. It is known that early and specific diagnosis of DHF or DSS followed by supportive therapy reduces morbidity and mortality [3].

Currently, the three basic methods used by most laboratories for the diagnosis of dengue virus infection are viral isolation, detection of the viral genomic sequence by a nucleic acid amplification technology assay (Reverse transcription-polymerase chain reaction (RT-PCR)), and detection of dengue virus-specific IgM antibodies by the IgM-capture enzyme-linked immunosorbent assay (MAC-ELISA) and/or the rapid dengue immunochromatographic test (ICT) [4].

Detection of NS1 has been a promising test to diagnose dengue in its early febrile stage due to its long half-life in the blood. The NS1 protein was found to be highly conserved in all dengue serotypes, circulating at high levels during the first few days of illness. It correlates with the development of DHF. There is no cross-reaction of the dengue NS1 protein with those of other related flaviviruses [5]. The present study was conducted to assess the correlation between platelet counts and serological markers of dengue infection.

\section{Materials and Method}

Setting: The present study was conducted in the Department of Microbiology and Pathology, GS Medical College and Hospital, Pilkhuwa, Dist. Hapur, Uttar Pradesh, India. It consisted of 120 patients suspected of dengue fever.

Data collection procedure: Data such as name, age, gender, etc. was recorded. Serum samples were collected from suspected dengue fever patients. The samples were tested for NS1 antigen, IgM, and IgG antibodies using the ICT test kit. The platelet count was recorded in dengue parameterpositive and -negative cases.

Statistical Analysis:Results were tabulated and subjected to statistical analysis. A P-value of less than 0.05 was considered significant.

\section{Results}

Table-1: Distribution of patients.

\begin{tabular}{|l|l|l|}
\hline \multicolumn{3}{|c|}{ Total- 120 } \\
\hline Gender & Male & Female \\
\hline Number & 70 & 50 \\
\hline
\end{tabular}

Table- 1 shows that out of 120 patients, males were 70 and females were 50 .

Table-2:Comparison of various dengue parameters.

\begin{tabular}{|l|l|l|}
\hline \multicolumn{1}{|c|}{ Parameters } & \multicolumn{1}{|c|}{ Number } & \multirow{2}{*}{ P-value } \\
\cline { 1 - 2 } NS1 only & 62 & \multirow{2}{*}{0.01} \\
\hline IgM only & 20 \\
\cline { 1 - 2 } IgG only & 14 \\
\cline { 1 - 2 } NS1 and IgM only & 10 \\
\cline { 1 - 2 } NS1 and IgG only & 8 & \\
\cline { 1 - 2 } IgM and IgG only & 6 & \\
\hline
\end{tabular}

Table-2 represents that NS1 only was detected in 62 , IgM only in 20, IgG only in 14, NS1 and IgM only in 10, NS1 and IgG only in 8 and IgM and IgG only in 6 cases. The difference was significant $(P<$ 0.05).

Table-3:Comparison of platelet count and dengue parameter.

\begin{tabular}{|c|c|c|c|}
\hline Parameters & |Number| & Platelet count $<1,00,000 / \mathrm{ml}$ & P-value \\
\hline NS1 only & 62 & 48 & \multirow{6}{*}{0.01} \\
\hline IgM only & 20 & 12 & \\
\hline IgG only & 14 & 10 & \\
\hline NS1 and IgM only & 10 & 6 & \\
\hline NS1 and IgG only & 8 & 4 & \\
\hline IgM and IgG only & 6 & 2 & \\
\hline
\end{tabular}

Table- 3 shows that 62 patients that were positive for NS 1, thrombocytopenia was seen in 48, 12 out of 20 IgM positive patients, 10 out of 14 IgG positive patients, 6 out of 10 NS1 and IgM only positive patients, 4 out of 8 NS1 and IgG only positive patients and 2 out of 6 IgM and IgG only positive patients were detected. The difference was significant $(P<0.05)$.

\section{Discussion}

Earlier case fatalities due to dengue infection have been reported to be around $2.5 \%$ to $5.4 \%$. Shock and organ impairment have been shown to be the most important factors that lead to fatalities in dengue infection. As a result of better fluid management regimes and greater awareness of associations of severe dengue and early interventions, the case fatality rates have signifi- 
Cantly dropped in many dengue-endemic countries. However, in order for early detection of those who are likely to develop severe dengue, the clinical and laboratory parameters are measured at least two or three times a day in all patients admitted to the hospital with dengue infection [7]. For a long time detection of dengue-specific IgG/IgM has been the mainstay of diagnosis of DI (Dengue Infection). The dengue-specific antibodies begin to appear only around the fifth day of fever in primary infection. Even in most secondary infections, both the IgM and IgG type antibodies cannot be recorded before the third day [8]. Therefore, there is always a window period, both in primary and secondary DI when only antibodies are tested. The new parameter, now available, for diagnosis of DI, the NS1 antigen, is detectable from day 1 of fever both in primary and secondary infections. It is important to note that NS1 is shown to be a highly specific viral marker making it an extremely reliable parameter for the diagnosis of DI from day 1 of the fever [9].The present study was conducted to assess the correlation between platelet counts and serological markers of dengue infection. In the present study, there were 70 males and 50 females. NS1 only was detected in 62 , IgM only in 20, IgG only in 14 , NS1 and IgM only in 10, NS1 and IgG only in 8 and IgM and IgG only in 6 cases.Paranavitaneet al10186 adult patients with confirmed dengue were enrolled during day 3-8 of illness. Clinical and laboratory parameters were recorded during the course of the illness and NS1 antigen levels were determined using both the Panbio dengue early ELISA and an NS1 rapid antigen detection kit. $59.1 \%$ of patients presented to the hospital on day 5-6 of illness when NS1 antigen positivity was significantly associated with severe dengue and the NS1 antigen levels were significantly higher in those who went on to develop shock. Serum NS1 antigen levels significantly and inversely correlated with the total white cell counts and lymphocyte counts. The bedside NS1 test showed comparable sensitivity (97.4\%) and specificity $(93.7 \%)$ to the laboratory NS1 test in our setting and cohort. It was found that 62 patients that were positive for NS 1 , thrombocytopenia was seen in 48,12 out of 20 IgM positive patients, 10 out of $14 \mathrm{IgG}$ positive patients, 6 out of 10 NS1 and IgM only positive patients, 4 out of $8 \mathrm{NS} 1$ and IgG only positive patients and 2 out of 6 IgM and IgG only positive patients were detected. Jyothyet al11 in their study a total of 520 serum samples were collected from the suspected dengue fever patients. Sixty-two samples tested positive for one or more denque-specific parameters. Out of the 62 samples,
39 (62.9\%)were positive for the NS1 antigen, only seven $(11.3 \%)$ were positive for IgM, and only three $(4.9 \%)$ were positive for IgG. A platelet count < $1,00,000 / \mathrm{ml}$ was observed in 32 cases $(51.6 \%)$. When the platelet count was done in 100 dengue parameter-negative fever patients (controls), thrombocytopenia was observed in $30 \%$ of the cases. Kulkarni et al [12]conducted a study in which serum samples from clinically suspected dengue cases were tested for NS1, IgM, and IgG by immunochromatography-based tests. Platelet counts were obtained for all positive cases and 150 dengue seronegative cases of fever that served as controls. Test results of dengue-specific parameters were compared against platelet counts. The proportions obtained were compared by Standard error of the difference between the proportions (SEP test). Of 2104 samples tested, 320 were positive for one or more dengue parameters. Of the 320,95 were positive for NS1 only, 161 showed IgM only while 9 showed IgG only. More than one marker was detected in the remaining 55 samples. Thrombocytopenia was more consistently associated whenever NS1 was detected compared to antibody detection. The inclusion of NS1 increases the detection rate of dengue fever. In cases of fever, thrombocytopenia is more consistently found in dengue positive rather than dengue negative subjects. It correlates well when NS1 and IgM are detected simultaneously.

\section{Limitations}

Since the study was conducted ata tertiary health center, patients reported after a few days of treat ment in the primary and secondary health care centers. More NS1-positive cases would have been detected if the test was done in the initial three to four days of fever. Further, the sample size is lessin the present study.

\section{Conclusion}

The authors concluded that in fever, thrombocytopenia is observed more uniformly in subjects positive for dengue. Whenever IgM is detected as compared to NS1 and can be used asan indicator to reduce the complication of dengue disease.

\section{What does this study add to the existing knowledge?}

The application of NS1 in the diagnosis of dengue 
Increases the detection rate significantly.

\section{Author's contributions}

Dr. Neha Goel: Contributed substantially to the conception, design, acquisition of data, analysis, and interpretation of data, drafting the article, critical revision of the material, and final approval of the version to be published. Dr. Ritika Kansal: Contributed substantially to the conception and design, critical revision of the article and, final approval of the version to be published. Dr. Amit Joon: Contributed substantially to the conception and design, acquisition of data, critical revision of the article, and final approval of the version to be published. Dr. Jaya T. Hemnani: Contributed substantially to the conception and design, acquisition of data, critical revision of the article, and final approval of the version to be published.

\section{Reference}

01. Gupta V, Yadav TP, Pandey RM, Singh A, Gupta $M$, Kanaujiya $P$, et al. Risk factors of dengue shock syndrome in children. J Trop Pediatr. 2011;57(6)451-456.

doi: [Article:https://doi.org/10.1093/tropej/fmr020] [Crossref]

02. Libraty DH, Young PR, Pickering D, Endy TP, Kalayanarooj S, Green S, et al. High circulating levels of the dengue virus nonstructural protein NS1 early in dengue illness correlate with the development of dengue hemorrhagic fever. J Infect Dis. 2002;186(8)1165-1168.

doi:[Article:https://doi.org/10.1086/343813]

[Crossref]

03. Guzmán MG, Kourí G. Dengue- An update. Lancet Infect Dis. 2001;2;33-42.

doi:[Article:https://doi.org/10.1016/s1473-3099(01)

00171-2][Crossref]

04. WHO. Dengue guidelines for diagnosis, prevention and control. In Southeast Asian Office of the WHO- World Health Organisation. 2009.

[Crossref]

05. Jayaratne S, Atukorale V, Gomes L, Chang T, Wijesinghe $T$, Fernando $S$, et al. Evaluation of the WHO revised criteria for classification of clinical disease severity in acute adult dengue infection. BMC Res Notes. 2012;5;645.

doi: [Article:https://doi.org/10.1186/1756-0500-5645][Crossref]
06. Malavige GN, Gomes L, Alles L, Chang T, Salimi $M$, Fernando $S$, et al. Serum IL-10 as a marker of severe dengue infection. BMC Infect Dis. 2013;13(1)341.

doi: [Article:https://doi.org/10.1186/1471-2334-13341][Crossref]

07. Lee LK, Gan VC, Lee VJ, Tan AS, Leo YS, Lye DC. Clinical relevance and discriminatory value of elevated liver aminotransferase levels for dengue severity. PLoSNegl Trop Dis. 2012;6(6)1676.

doi:[Article:https://doi.org/10.1371/journal.pntd. 0001676][Crossref]

08. Malavige GN, Huang LC, Salimi M, Gomes L, Jayaratne SD, Ogg GS. Cellular and cytokine correlates of severe dengue infection. PLoS One. 2012;7(11)50387.

doi:[Article:https://doi.org/10.1371/journal.pone. 0050387][Crossref]

09. Tricou V, Vu HT, Quynh NV, Nguyen CV, Tran HT, Farrar J, et al. Comparison of two dengue NS1 rapid tests for sensitivity, specificity and relationship to viraemia and antibody responses. BMC Infect Dis. 2010;10;142.

doi: [Article:https://doi.org/10.1186/1471-2334-10142][Crossref]

10. Kulkarni RD, Patil SS, Ajantha GS, Upadhya AK, Kalabhavi AS, Shubhada RM, et al. Association of platelet count and serological markers of dengue infection- importance of NS1 antigen. Indian J Med Microbiol. 2011;29;359-62.

doi: [Article:https://doi.org/10.4103/02550857.90159][Crossref]

11. Paranavitane SA, Gomes L, Kamaladasa A, Adikari TN, Wickramasinghe N, Jeewandara C, et al. Dengue NS1 antigen as a marker of severe clinical disease. BMC Infect dis. 2014;14(1)570.

doi: [Article:https://doi.org/10.1186/s12879-0140570-8][Crossref]

12. Jyothi P, Metri BC. Correlation of serological markers and platelet count in the diagnosis of Dengue virus infection. AdvanBiomed Res. 2015;4;26.

doi:[Article:https://doi.org/10.4103/2277-

9175.150396][Crossref] 